\title{
Study of Cataclysmic Variables with the Satellites LOFT and Gaia
}

\author{
R. Hudec ${ }^{1,2}$, V. Šimon ${ }^{1,2}$ \\ ${ }^{1}$ Astronomical Institute, Academy of Sciences of the Czech Republic, CZ-25165 Ondřejov, Czech Republic \\ ${ }^{2}$ Czech Technical University in Prague, Faculty of Electrical Engineering, Prague, Czech Republic
}

Corresponding author: rene.hudec@asu.cas.cz

\begin{abstract}
The goal of this paper is to discuss the capabilities of ESA satellite missions Gaia (already in space) and LOFT (considered for the ESA M4 slot) for investigation of cataclysmic variables (CVs). Both Gaia and LOFT can contribute to study of $\mathrm{CVs}$ and related objects. Spectrophotometry and low dispersion spectroscopy are the most important for CV analyses with the observations of Gaia. We present the possible strategies for investigation of CVs in the sampled photometric and spectroscopic data provided by Gaia. E.g. statistical properties of the long-term activity of various types of CVs can be determined from them. LOFT can be a promising satellite to provide a sensitive X-ray monitor which will enable to investigate the little studied long-term activity of various types of CVs (especially the magnetic ones) in the X-ray band.
\end{abstract}

Keywords: cataclysmic variables - dwarf novae - intermediate polars - X-rays - LOFT - Gaia.

\section{Introduction}

Although two ESA satellite missions, namely Gaia (already in space) and LOFT (considered for the ESA M4 slot), focus on different science areas, both of them can effectively contribute to the investigation of cataclysmic variables $(\mathrm{CVs})$.

\section{The ESA Gaia Mission}

The ESA satellite Gaia was successfully launched on December 19, 2013. Gaia is an ambitious mission to chart a three-dimensional map of our Galaxy, in the process revealing the composition, formation and evolution of the Galaxy (e.g. de Boer et al. 2000 and Eyers et al. 2013). Gaia will provide unprecedented positional and radial velocity measurements with the accuracies needed to produce a stereoscopic and kinematic census of about one billion stars in our Galaxy and throughout the Local Group. This amounts to about 1 percent of the Galactic stellar population.

\subsection{Astrophysics with Gaia}

Motivation of this paper is to outline the possibilities of performing astrophysics of CVs with Gaia data. This is not trivial as the main goal of the Gaia mission is to create a catalog. The photometric sampling provided by Gaia will not be optimal for many variable astrophysical sources. However, the fine spectrophotometry (in reality ultra low resolution spectroscopy) provided by $\mathrm{BP} / \mathrm{RP}$ photometers will be unique and important for many astrophysical investigations with Gaia, including cataclysmic variables science (Hudec \& Šimon 2007a and 2007b; Hudec et al. 2012 and 2013).

Even the sampled Gaia data can provide the following properties of the object:

1. Determination of amplitude of the brightness variations

\section{Statistical distribution of brightness}

3. Absolute magnitude (from distance determined from parallax (and interstellar extinction)). Note that these items 1, 2, and 3 even enable to establish the physically justified sequence of types of CVs.

4. Color indices and their time variations

In principle, it is also possible to search for the periods and cycles of the brightness variations, but one has to very cautious in doing this procedure using the sampled data from Gaia.

The sampling of the data provided by Gaia is not optimal for the astrophysical work; these observations are not dense enough and not equidistantly distributed. Typically, one can expect 50-100 photometric points over 5 years. However, additional data can be provided by the ground-based experiments.

We propose the following two approaches for classification and verification of CVs in the sampled Gaia data. The first one is to search for outbursts or high/low 
state transitions, i.e. analysis of parameters of the light curve not strongly dependent on sampling.

\subsection{Statistical properties of the long-term activity of CVs, perspectives of histograms for Gaia}

We investigated the profiles of the light curves of CVs of various types with real observations (daily means) from the AFOEV database (http://cdsarc.ustrasbg.fr/afoev/). We approximated the Gaia sampling of these light curves by selecting the data separated by $\sim 20$ days. This procedure yielded the amount of the sampled data and the length of the mapped time segment which roughly corresponded to the expected time of observing with Gaia. We investigated which methods can yield correct identification of a CV of a given type almost independent of the sampling. We identified the impact of the Gaia data on the investigation of CVs which are very active objects.

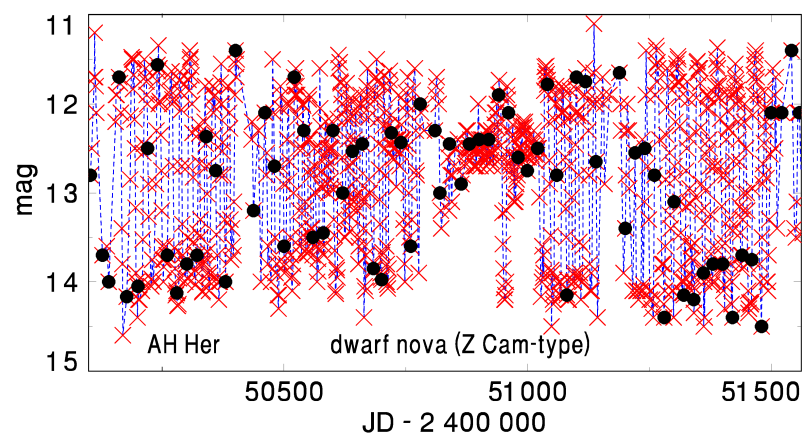

Figure 1: Comparison of the observed (one-day means of AFOEV data (crosses)) and the approximated sampled data of the dwarf nova AH Her (closed circles). Heavily sampled data are expected from the Gaia satellite. Notice that it is difficult to resolve the profile of the light curve in the sampled data.

The basic profile of the high state and its fluctuations in e.g. novalike systems and supersoft $\mathrm{X}$-ray sources can be plausibly mapped by the sampled Gaia data. Only the high state/low state transitions may be missed or covered by only one or two data points by Gaia. On the other hand, we expect the large outbursts of a dwarf nova to be covered by only a very few data points while short outbursts may be missed in the Gaia data set. Moreover, the individual outbursts will be probably captured in different phases due to their short duration. Gaia will therefore usually not provide us with information about the profile of the outburst. However, we found that the statistical distribution of magnitudes of a given CV is only slightly distorted if a long time (several years) interval is covered. The statistical distribution of brightness and its parameters like the standard deviation, skewness, excess are a representative description of the properties of the long-term activity of CVs. These histograms are only slightly distorted by the sampling of the Gaia observations if a long (several years) time segment is covered by the observations. Nevertheless, a distortion may appear if the recurrence time of outbursts is close to the sampling time of Gaia.

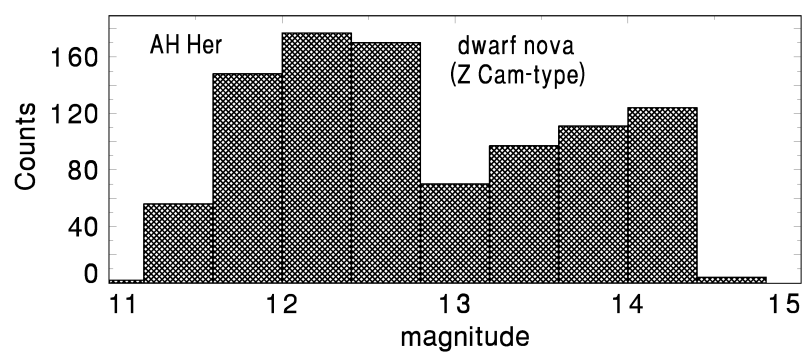

Figure 2: Statistical distribution of the observed data in the dwarf nova AH Her (from Fig. 1).

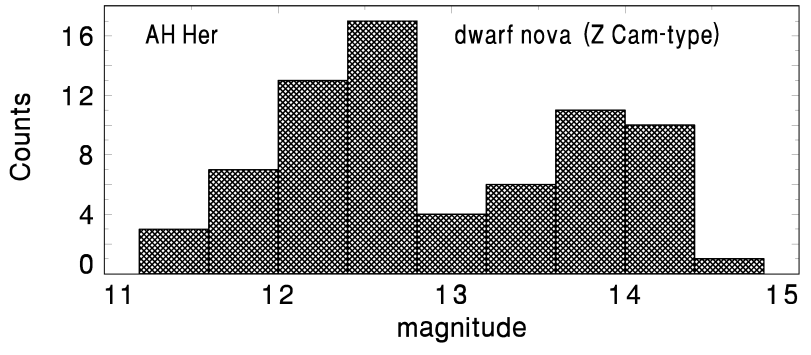

Figure 3: Statistical distribution of the approximated sampled Gaia data of the dwarf nova AH Her (from Fig. 1). Notice that the histogram is not dramatically influenced by the sampling and is similar to that of the observed data.

We conclude that the sequence of the types of CVs, justified by the physics of accretion onto the compact objects, is reflected in the statistical distribution of the long-term brightness variations, hence it is suitable for analysis of the sampled Gaia data. The importance of this sequence can be even enhanced if the apparent magnitudes are transformed to the absolute magnitudes by using the distances of CVs determined from the parallaxes.

\subsection{Rare flares (different from outbursts in dwarf novae) in CVs}

We investigated the possibility to detect and identify rare phenomena in CVs with Gaia. Deep monitoring of a large number of objects can lead to discoveries of more CVs with flares (phenomena different from outbursts) like the event discovered by van Amerongen 
\& van Paradijs (1989) and to detection of additional such events even in the already known systems. We investigated some CVs in Bamberg photographic plates which have a similar sampling and coverage as the expected Gaia data. We conclude that the discovery of such events is possible with the monitoring planned for Gaia (Šimon 2010).

\subsection{Ultra-low dispersion spectra}

The ultra-low dispersion spectra will be provided by Gaia RP/BP photometers. They can play a significant role in (1) searches for prominent spectroscopic variability, and (2) searches for objects with prominent (maybe variable) spectral features.

As an example, the spectra of the outburst of V407 Cyg (Munari et al. 2011) (or of an analogous object) could be investigated by Gaia. The spectral appearance of V407 Cyg in outburst was a highly peculiar one. The spectrum of this event is completely different from those ever recorded for this object and other symbiotic Mira variables. The white dwarf companion to the Mira variable experienced an outburst similar to that of classical novae, and its ejecta were moving in the circumstellar environment already filled by the ionized wind of the Mira.

We summarize several types of CVs and the related systems with very bright emission lines:

- Supersoft X-ray sources in the optical high states (Steiner \& Diaz 1998). Their spectra display intense and broad emission lines (especially $\mathrm{H} \alpha$ ) (e.g. V Sge (Herbig et al. 1965)). Such systems also often have Balmer jump in emission.

- Classical novae in the nebular phase of the outburst (late phase, weeks to months after the maximum of the optical luminosity) - faint continuum, very strong emission lines of various elements (e.g. Munari et al. 2013).

- Symbiotic systems: very strong emission lines (mainly $\mathrm{H} \alpha$ ) (e.g. Davidson et al. 1978).

- Dwarf novae: highly variable strong Balmer jump (e.g. Walker \& Chincarini 1968).

\subsection{The color indices from RP/BP spectra}

The color indices can be determined from the spectra obtained by the Gaia RP/BP photometers. Significant results are expected to be immediately available, there is no need to wait for years as in the photometry case.

The color indices give important information on the spectral energy distribution. These indices can play an important role in classification and/or verification of CVs. They are also important for a search for the common properties of the sources of a given kind (e.g. to help identify a source as a CV and/or to study the evolution of the spectral profile with time as the CV undergoes various states of its activity). Not only the color indices of the object at a given time, but also the time evolution of these indices are therefore important. They can also play a role in resolving among the individual radiation mechanisms (e.g. cyclotron radiation versus thermal emission). Even variations of strong emission lines with respect to the continuum can be resolved by the color indices (e.g. $\mathrm{H} \alpha$ changes, changes of Balmer jump between emission and absorption). The color indices are also important for forming a representative ensemble of events (e.g. outbursts) in a given CV or in a given type of CVs. This is possible also for faint objects detected by Gaia (down to mag $\sim 20$ ).

The parallaxes from Gaia will enable to determine the distances, and hence the absolute magnitudes of many CVs. It will be therefore possible to determine and study the relation between the color indices and the absolute magnitudes of CVs of various types.

\subsection{Searches for cycles or periods}

Variograms allow us to search for characteristic timescales or quasiperiods which extend just for several epochs of the cycle. Variogram characterizes the spatial continuity or roughness of a data set. Variograms are important for a search for the superorbital cycles in the long-term activity of CVs and low-mass X-ray binaries because their long-term activity is not periodic. Standard period searches therefore often reveal nothing.

We used the light curves of CVs of various types for testing. We applied the real observations from the AFOEV database for feasibility study of Gaia data. We approximated the Gaia sampling of these light curves by the data separated by $\sim 20$ days. We investigated how the variograms were modified by the sampling. An example, the supersoft X-ray source V Sge, is displayed in Fig. 4. The amount of the sampled data and the length of the mapped time segment roughly correspond to the expected time of observing of Gaia. Since the duration of the high or the low state is longer than the time interval between the sampled data, the profile of the light curve is still recognizable.

Our tests showed that, in the case of the Gaia data, variograms are suitable especially for investigation of the types of CVs which display the light curves, whose profiles are gradual. V Sge with its alternating high and low states and the amplitude of the brightness variations similar for the individual epochs of the cycle is a good example (Figs. 5 and 6). Similar results can be obtained for novalike CVs with the episodes of the high and low states. However, it is necessary to be cau- 
tious in the case of dwarf novae because the outbursts, although with a large amplitude, are usually considerably shorter than the intervals of quiescence. This implies that some outbursts may be missed in the sampled data. It also emerged that binning of the data for variograms (Fig. 6) plays a big role. Fine binning yields a more precise determination of the cycle-length, but artifacts and false detections can appear, especially if the data are sampled.

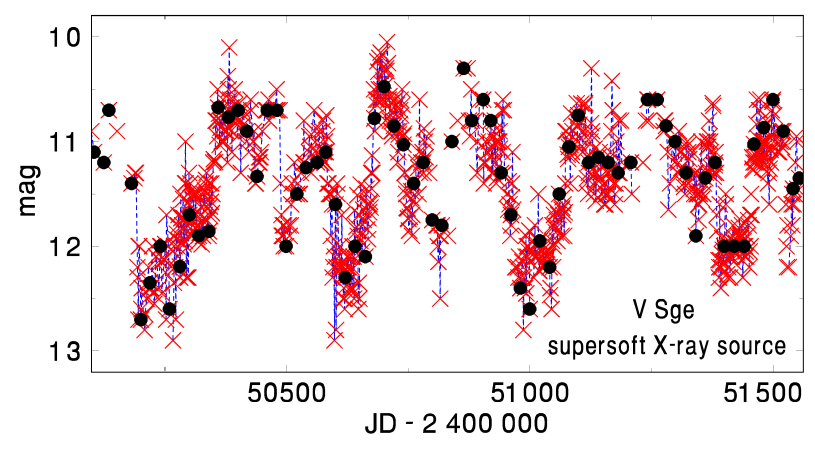

Figure 4: Comparison of the observed (one-day means of AFOEV data (crosses)) and the approximated sampled data (closed circles) of the supersoft X-ray source V Sge. Heavily sampled data are expected from the Gaia satellite.

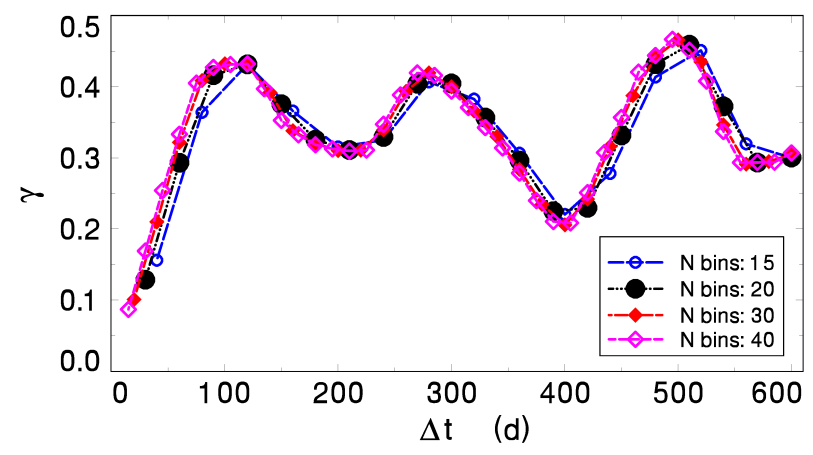

Figure 5: Variogram for the observed data of the supersoft X-ray source V Sge (from Fig. 4). N bins refers to the number of bins used for constructing the variogram.

The orbital modulation of CVs can be searched for and investigated in the sampled Gaia data only under some favorable circumstances. Although the length of the orbital period of $\mathrm{CV}$ can be considered to be stable (or at most slightly variable) during the proposed lifetime of Gaia, it is reasonable to expect that the orbital modulation will be influenced by the long-term variations (e.g. outbursts, episodes of the high and low states). The amplitude of the long-term variations can often be considerably larger than that of the orbital modulation even in the case of a significant amplitude of this modulation (e.g. $>0.8 \mathrm{mag}$ ). Only if the long-term level of brightness remains almost stable (or if the limited segments of the long-term light curve are selected), the orbital modulation can emerge. A preferable situation will be to fold the Gaia data with the already known orbital period, and to investigate if and how the profile of the modulation varies with the changes between the states of activity. The orbital period can be determined for example by the follow-up observations of the ground-based telescopes. This is one of the cases of co-operation of the orbital and the ground-based observing components of the project.

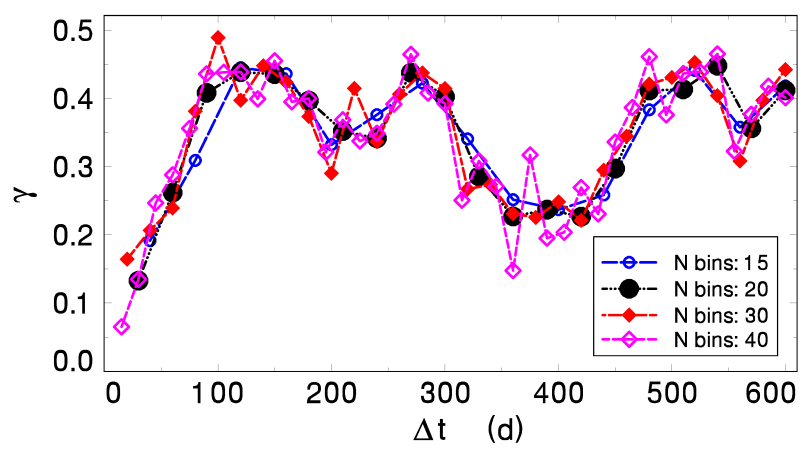

Figure 6: Variogram for the approximated Gaia data of the supersoft X-ray source V Sge (from Fig. 4). A higher value of $\mathrm{N}$ bins can yield a better determination of the possible cycle-length, but also a bigger noise.

\section{ESA LOFT}

LOFT (the Large Observatory For x-ray Timing) is specifically designed to exploit the diagnostics of very rapid X-ray flux and spectral variability in compact objects, yielding unprecedented information on strongly curved spacetimes and matter under extreme conditions of density and magnetic field strength (Feroci et al. 2012; Brandt et al. 2013). LOFT is designed to investigate variability from submillisecond quasi-periodic oscillations (QPO) to years long transient outbursts.

LOFT belongs to ESA Medium Class mission (M3 candidate) and was selected in Feb 2011 to complete a 3year Phase 0/A study. Final down selection on Jan 2014 (5 competed for single launch slot) was however negative for LOFT. There is however a chance to be selected for M4.

LOFT is observatory-type mission: there are 2 onboard instruments and science data center provided by the community. The originally expected launch date was 2022-2024 timeframe (Soyuz launcher) with 4+1 years mission lifetime and Low Earth orbit $(550 \mathrm{~km}$, 2 ground stations, $7 \mathrm{~Gb}$ science data/orbit).

The LOFT Large Area Detector (LAD) has an effec- 
tive area $\sim 20$ times larger than any largest predecessor, uniquely combined with a CCD-class energy resolution. LOFT LAT is suitable for observing CVs in a wide $\mathrm{X}$ ray band of $1-40 \mathrm{keV}$, with very fine time resolution.

The LOFT Wide Field Monitor (WFM) has a 4 steradian field of view at soft X-rays to discover and localize X-ray transients and impulsive events and to monitor spectral state changes, triggering follow-up observations and providing a wealth of science in its own. LOFT WFM is suitable for monitoring of the outbursts and high states of CVs in the energy range of $1-50 \mathrm{keV}$.

\subsection{The LOFT science}

The main $L O F T$ science is the study of neutron star structure and equation of state of ultradense matter $(3+$ independent methods): (1) neutron star (NS) mass and radius measurements, and (2) neutron star crust properties. In addition to that, strong gravity and the mass and spin of black holes will be investigated (5 independent methods), e.g. quasi-periodic oscillation evolution, and, in the time domain, nFe line tomography and reverberation studies in bright active galactic nuclei (AGNs) and black hole candidates (BHCs) ((also vs NSs), and relativistic precession. LOFT Observatory Science is expected to provide important observations for virtually all classes of relatively bright sources, including: X-ray bursters, High-mass X-ray binaries, Xray transients (all classes), CVs, magnetars, gamma-ray bursts (GRBs), Nearby galaxies (SMC, LMC, M31...), AGN etc.

In general, CVs are X-ray emitters (e.g. Warner 1995). However, the properties and time evolution of their X-ray spectra strongly depend on the type and the state of activity of a given CV. Although some general relations exist for a given $\mathrm{CV}$ type, each $\mathrm{CV}$ is really specific in this regard. Since the proposed band of LOFT is $1-50 \mathrm{keV}$, those CVs with the hardest Xray spectra will be the suitable targets for observing with this satellite. For example, the intermediate polars usually display the significantly larger values of $\mathrm{k} T$ in comparison with non-magnetic CVs (Šimon et al. 2006).

Magnetic CV often have the hard X-ray intensity correlated with the mass accretion rate onto the white dwarf because of the accretion rate onto the magnetic poles of the WD. The dwarf nova outbursts of intermediate polars can be therefore accompanied by a strong increase of the hard X-ray intensity (e.g. DO Dra (Szkody et al. 2002), GK Per (Fig. 7)). In polars (e.g. Warner 1995), the hard X-ray intensity strongly increases in the optical high states. However, the case of the polar AM Her shows that the relation of the optical and hard X-ray emission varies for the individual episodes of the high state (Šimon 2011). This empha- sizes the necessity to obtain the observations of a larger ensemble of CVs to study the processes and their parameters which influence the behavior of $\mathrm{CVs}$ on the long timescales. We therefore expect the satellites like $L O F T$ to be important instruments for investigation of magnetic CVs.

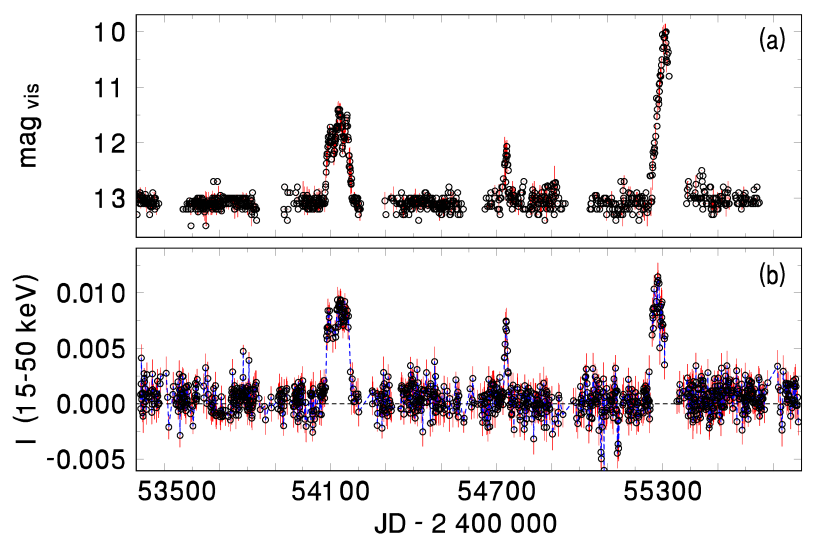

Figure 7: Outbursts of the intermediate polar GK Per in the optical band (AFOEV data) (a) and the hard Xray band (BAT/Swift data (data of Krimm et al. 2013).

\section{Conclusions}

Both Gaia and LOFT can contribute to study of CVs and related objects. Spectrophotometry and low dispersion spectroscopy are the most important for $\mathrm{CV}$ analyses with the observations of Gaia. Both the LOFT WFM X-ray monitoring in the energy range of 1-50 keV and detailed LAD observations with fine time resolution are important in this research field. Gaia was launched on December 19, 2013. LOFT was not selected in competition with other ESA M3 candidates, but it remains a promising candidate for M4.

It is true that dense series of observations covering the intervals of several years are necessary to investigate the rising and decaying branches of outbursts and high/low states. Nevertheless, a properly chosen strategy can yield suitable results of analyses even in the sampled photometric and spectroscopic data provided by Gaia. We propose to obtain the statistical properties of the long-term activity of a representative ensemble of events (e.g. outbursts, high and low states) in a very large ensemble of a given type of systems. This is important for our understanding of the physical processes involved in the systems distributed in various regions of the Galaxy which may have a different history of the star formation and chemical evolution.

We also argue in favor of the development of more sensitive X-ray monitors that will be able to detect the long-term activity of CVs. Even the available short data series obtained by the pointed X-ray observations 
of some known CVs revealed the very large variety of properties of their X-ray emission (e.g. Warner 1995). The current monitors are able to observe mainly the luminous binary X-ray sources which contain the NS or the BH. Since only a very few CVs are detectable by these instruments, LOFT can be a promising satellite to provide a sensitive X-ray monitor.

\section{Acknowledgement}

We acknowledge partial support by GA CR grants 1339464J and 13-33324S. We also acknowledge the use of public data from Swift/BAT transient monitor provided by the Swift/BAT team. We also used the observations from the AAVSO International database (Massachusetts, USA) and the AFOEV database operated in Strasbourg, France.

\section{References}

[1] Brandt, S., et al., 2013, EAS Publications Series, Volume 61, 2013, pp.617-623

doi:10.1051/eas/1361098

[2] Davidson, K., et al., 1978, ApJ, 220, 239

[3] de Boer,K., Gilmore, G., Hog, E., Lattanzi, M.G., Lindegren, L., Luri, X., Mignard, F., Face, O., Ferryman, M., de Zeeuw, P.T. (eds) GAIA Concept and Technology Study Report (the 'Red Book') ESA-SCI(2000)4 pp381 (ESA Paris) 2000

[4] Hudec, R. et al., 2013, Acta Polytechnica, Vol. 53 , No. 3 , p.30

[5] Eyer, L. et al., 2013, Central European Astrophysical Bulletin, p. 115-126

[6] Feroci, M. et al., 2012, Experimental Astronomy, Volume 34, Issue 2, pp.415-444 doi:10.1007/s10686-011-9237-2

[7] Herbig, G. H., et al., 1965, ApJ, 141, 617 doi:10.1086/148149
[8] Hudec, L., 2007, Algorithms for spectral classification of stars, BSc. Thesis, Charles University, Prague

[9] Hudec, R., Šimon, V., 2007a, Specific object studies for cataclysmic variables and related objects ESA Gaia Reference Code GAIA-C7-TN-AIO-RH-001-1.

[10] Hudec, R., Šimon, V., 2007b, Specific object studies for optical counterparts of high energy sources. ESA Gaia Reference Code GAIA-C7-TN-AIO-RH-002-1. doi:10.14311/AP. 2013.53.0799

[11] Hudec, R., Šimon, V., Hudec, L., 2013, Acta Polytechnica, Vol 53, Supplement, p.798 doi:10.1088/0067-0049/209/1/14

[12] Krimm, H. A., et al., 2013, ApJS, 209, 14 doi:10.1111/j.1745-3933.2010.00979.x

[13] Munari, U., et al., 2011, MNRAS, 410, L52 doi:10.1093/mnras/stt1340

[14] Munari, U., et al., 2013, MNRAS, 435, 771

[15] Šimon, V., Mattei, J. A., 1999, A\&AS, 139, 75

[16] Šimon, V., 2000, A\&A, 360, 627

[17] Šimon, V., et al., 2006, IAUS, 230, 66

[18] Šimon, V., Hric, L., Petrík, K., et al., 2002, A\&A, 393, 921

[19] Šimon, V., 2010, Advances in Astronomy, article id. 382936 doi:10.1016/j .newast. 2011.03.001

[20] Šimon, V., 2011, NewA, 16, 405

[21] Steiner, J. E., Diaz, M. P., 1998, PASP, 110, 276

[22] Szkody. P., et al., 2002, AJ, 123, 413

[23] van Amerongen, S., van Paradijs, J., 1989, A\&A, 219,195

[24] Walker, M., \& Chincarini, G., 1968, ApJ, 154, 157 